\title{
Integrated Dual-Channel X-Band Offset-Transmitter for Phase Steering and DDMA Arrays
}

\author{
Johan C. J. G. Withagen ${ }^{1}$, A. J. Annema ${ }^{1}$, B. Nauta ${ }^{1 \cdot}$, F. E. van Vliet ${ }^{1 .} 2$. \\ 1. University of Twente, EWI/ICD, Enschede, 7500 AE, Netherlands, +31 53486 2644, j.c.j.g.withagen@utwente.nl \\ 2.TNO, The Hague, 2509 JG, The Netherlands
}

\begin{abstract}
Multiple-Input and Multiple-Output (MIMO) arrays provide more degrees of freedom than conventional phased arrays. They require that every transmitting element of the array can be identified when received. One way to achieve this is to give every element its own unique frequency. Offset-Transmitters may be used to introduce MIMO or Doppler Division Multiple Access (DDMA) into phased-arrays without an excessive increase in waveform-generating hardware. Our dual-channel demonstrator IC can obtain a phase accuracy better than 1 degree and an spurious level of better than $-65 \mathrm{dBc}$ for a single on-chip channel. This work investigates at X-band the effects of the limited on-chip isolation of $35 \mathrm{~dB}$, when multiple offset outputs are generated on a single chip for both beam steering and DDMA. In case of beam steering, the requirements on channel-to-channel isolation are less strict, making it well within reach. In the case of DDMA, we recommend increasing the channel-to-channel isolation by implementing multiple chips, in which case independent signals can be generated.
\end{abstract}

Index Terms-Radar, BICMOS intgrated ciruits, MIMO

\section{INTRODUCTION}

Traditionally, phased arrays are used predominantly in radar systems. Recently however, phased arrays are emerging in many other applications, such as medial scanners and communication systems. The main benefit of phased arrays is the ability to change the directivity of the antenna array by electronically manipulating phase shifts in each array element. This is known as beam forming and can be performed both in receive and transmit elements.

In the last couple of decades the trend for the receive path is that the signals from every array element are digitized and that phase shifts are applied in the digital domain. This allows for the generation of multiple beams simultaneously on receive at the cost of processing power. This greatly increases the flexibility of beam forming in the receive path in comparison with more traditional analogue phase shifters.

The transmit elements, however, have largely remained based on traditional analogue phase shifters. In this way, the highquality signal generation does not need to be duplicated for every transmit element. The disadvantage, however, is that the transmitted signal at every antenna element is identical except for a phase shift.

In emerging technologies such as Multiple-Input and MultipleOutput (MIMO) transceivers [1], 5G [2] and Multi-Carrier Modulation more flexibility is required in the transmit elements. In MIMO every transmitting element in the array transmits a unique waveform that can uniquely be identified in the receive chain. This inherently allows beam forming both in the transmit and in the receive path at cost of digital processing power, as shown in [3]. This approach significantly increases the flexibility of the total system. To be able to uniquely identify transmit array elements in the receive path, each element needs to transmit orthogonal waveforms. In this paper the per-transmit element orthogonality is achieved by transmitting unique nearby spaced frequencies per antenna element. This mechanism is denoted as Doppler Division Multiple Access (DDMA), [4], [5].

MIMO systems have several benefits in array applications. It can mitigate RF array impairments as described in [6]; spurious responses, phase noise and other non-idealities are added incoherently in MIMO arrays where they typically add coherently in conventional phased arrays. Besides the increased flexibility to generate beams on both transmit and receive and the inherent better robustness with respect to e.g. power amplifier non-idealities, MIMO arrays have a better angular resolution when compared to similar size traditional phased arrays [3].

In this paper, orthogonal signals in each of the $N$ MIMO array elements are implemented as $N$ unique narrowly spaced tones. These tones are created from a very accurate shared RF Local Oscillator (LO), shifted in frequency (or phase) by mixing with a low-frequency Baseband (BB) signal that is unique for each array element. This mixer is denoted as Offset-Transmitter (OT). A top-level schematic of a MIMO transceiver using $N$ OTs to drive $N$ array elements is shown in Fig. 1.

OTs ideally only shift a frequency $f_{\mathrm{LO}}(t)$ by a frequency $\Delta f$ or phase shift $\Delta \varphi$. Actual OT circuits introduce many nonidealities; in the context of MIMO the most important ones include generating spurious tones, generating noise, source pull effects, coupling effects and cross-talk between any of the $N$ OTs in the array.
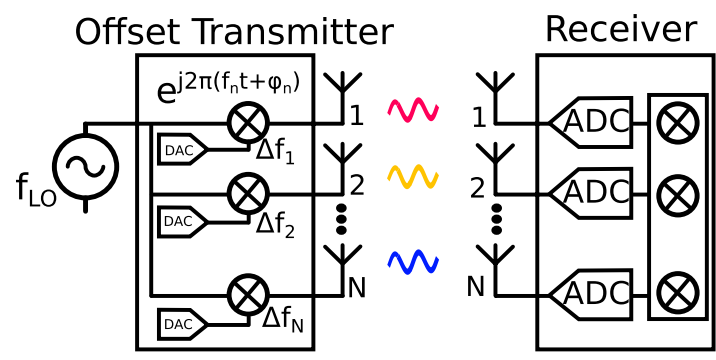

Fig. 1: An array with $N$ elements splitted in transmit and receive. On transmit, each element can offset a common RF signal $f_{\mathrm{LO}}(t)$ by a phase offset or (here) frequency offset $\Delta f_{n}$ created by an offset transmitter. At receive, each input signal at each element is digitized after being downconverted before processing (not shown). 
This paper focuses on the impact of non-ideal OTs on MIMO array performance. For this, we use our previously designed dual-channel OT IC in a minimum array configuration as demonstration vehicle. The single-channel performance and the low-frequency calibration loop that linearizes each OT in this IC are already presented in [7]. Section II briefly reviews our dual-channel OT system and its non-ideal properties that may impact array performance. Beam forming and MIMO require phase offsets respectively frequency offsets between array elements. Sections III and IV present analysis of the impact of OT non-idealities on the array performance. Finally, in section $\mathrm{V}$ the conclusions are discussed.

\section{INTEGRATED OFFSET TRANSMITTER}

Fig. 2 shows our dual-channel OT frontend, [7], from a high level point of view. The IC contains two linearized I/Q mixers that can offset an LO in $\mathrm{X}$-band frequencies by $\Delta f$ up to $\pm 2 \mathrm{MHz}$ or by an arbitrary fixed phase with an output power of $-21 \mathrm{dBm}$. Calibration was shown to be able to suppress inband spurs to below $-73 \mathrm{dBc}$ for single channel operation which is sufficient to create sufficiently orthogonal signals in MIMO arrays. For dual-channel operation purposes, each channel has its own Poly Phase Filter (PPF) to create I and Q signals from the externally generated X-band LO (between 8 and 10GHz). Having two separate per-channel PPFs significantly reduce mutual source pulling effects between the two channels.

The BB signals that determine the frequency or phase offset to be created by the OTs are generated by external BB Arbitrary Waveform Generators (AWGs) that drive the relatively high ohmic BB inputs of the dual-channel OT chip.

The IC was implemented in a $250 \mathrm{~nm}$ SiGe BiCMOS process. For the IQ-mixers in each OT the main advantage of this technology is that the flicker noise and flicker noise corner frequency are very low which allows for implementing small (frequency and/or phase) offsets with low phase noise.

The die micrograph is shown in Fig. 3; it measures $2.1 \mathrm{~mm} \mathrm{x}$ $2.1 \mathrm{~mm}$. The channels are placed perpendicular to one another to reduce on-chip coupling and pulling effects.

For MIMO application, the signals transmitted per array element must be orthogonal in order to be able to uniquely identify them in the receive chain. The dominant effects in

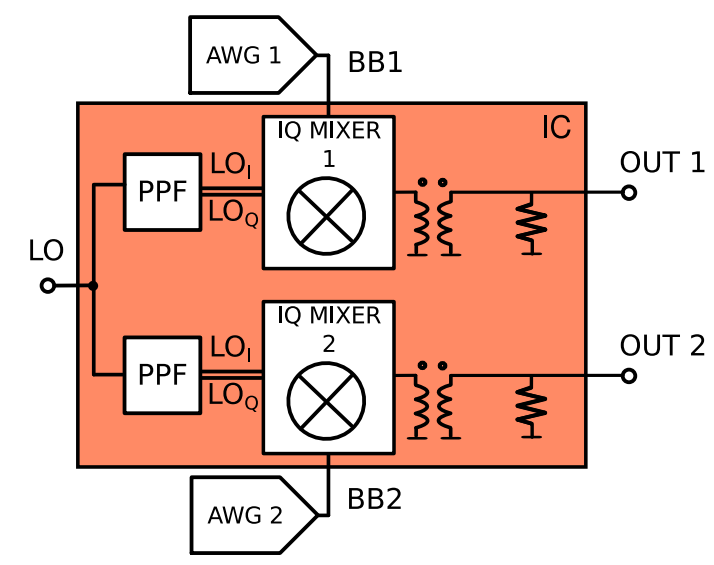

Fig. 2: The block schematic of the 2-channel Offset Transmitter.

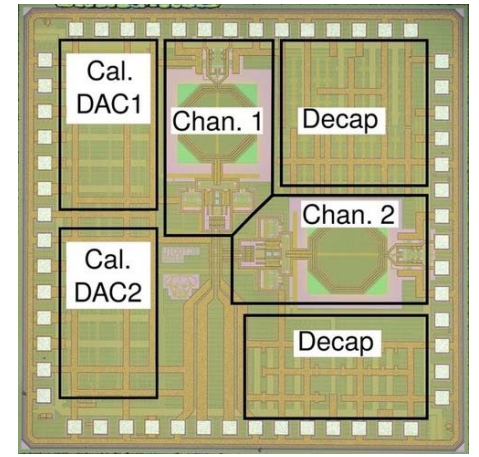

Fig. 3: Die micrograph; the BiCMOS chip measures $2.1 \mathrm{~mm} x$ $2.1 \mathrm{~mm}$.

OTs that hamper this identification capabilities are

- channel linearity, as this may cause in-band spurs that severely compromise the element identification in receive

- on-chip channel-to-channel isolation, as this directly reduces the orthogonality of transmit signals, and consequently reduce MIMO capabilities. If the two channels in a single chip generate different frequencies, as required by MIMO, the channel-to-channel isolation should be high enough, to prevent time dependent phase errors and unwanted inter-modulation products.

- chip-to-chip isolation, same issues as above

- noise, same issues as above.

In our dual-channel OT, calibrated channel linearity and noise performance are sufficient for many MIMO applications. However, the on-chip channel-to-channel isolation and chip-to-chip isolation limit MIMO capabilities which is the subject of the next two sections in this paper.

For our measurements, a PCB was implemented in a multilayer Rogers board. On this PCB, the output of the two channels of the dual-channel OT IC are perpendicular and implemented as strip lines to minimize coupling. Additionally, RF shielding was placed around the IC to further suppress coupling.

\section{IMPACT OF OT-LIMITATIONS ON BEAM STEERING CAPABILITIES OF ARRAYS}

Fig. 4 shows a small ( $4 \times 2$ patch) antenna array configuration assumed to derive the impact of OT imperfections on array performance. In the vertical plane, array elements are driven at

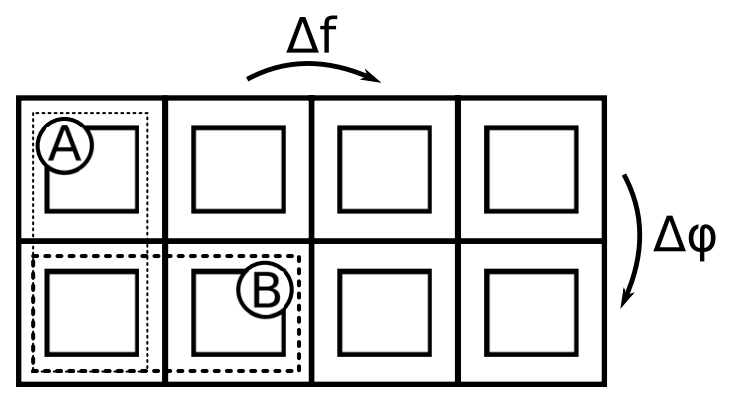

Fig. 4: Example of a $4 \times 2$ patch antenna array configuration. Per two vertical antenna-elements, a single chip with two offset transmitters was realized. The two evaluation experiments cover on-chip (A) phase steering and DDMA (B). 


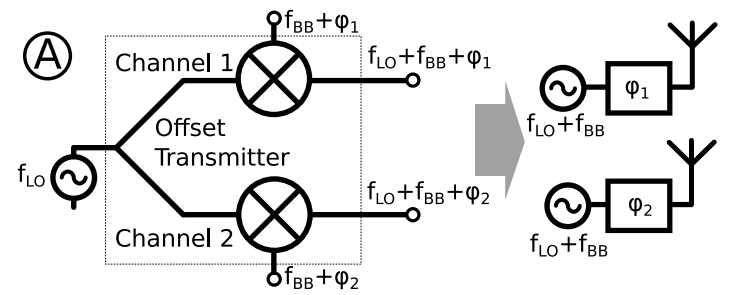

Fig. 5: The dual-channel Offset Transmitter (left) to implement phase-steering (right); the resulting antenna pattern is fixed for a given $\varphi_{1}$ and $\varphi_{2}$.

fixed phase offsets in a beam forming manner. Array elements in the horizontal plane are driven at different frequencies in a MIMO manner, [8]. A minimum array configuration is for these are indicated by A respectively B in Fig. 4.

Two vertical patch antennas, with only a phase difference, can be driven by a dual-channel OT chip, discussed in section II. Channel-to-channel isolation is about $35 \mathrm{~dB}$, from simulations and measurements. The lack of isolation causes a static phase error between the channels, with a maximum phase phase error when the phase difference between the two channels is $0.5 \pi$. Eqn. (1) shows that an isolation of $35 \mathrm{~dB}$, yields a phase error of maximum of $1^{\circ}$, which for most phased array applications is well within the arrays requirements.

$$
\epsilon_{\varphi}=\tan ^{-1}(-35 \mathrm{~dB}) \approx 1^{\circ}
$$

In Fig. 5, a simplified measurement setup is shown. A dualchannel OT IC is used for beam steering. Two external AWGs provide two signals with the same frequency $\mathrm{f}_{\mathrm{BB}}$ at a phase offset $\Delta \varphi$. AWG1 generates $f_{\mathrm{BB}}+\varphi_{1}$, while AWG2 generates $f_{\mathrm{BB}}+\varphi_{2}$; both signal are upconverted with $f_{\mathrm{LO}}$. Circulators are placed at the outputs of channel 1 and 2, for measurement purposes. These provide additional reverse isolation to prevent load pulling. In a actual array implementation, these are to be replaced by power amplifiers, that provide isolation from load

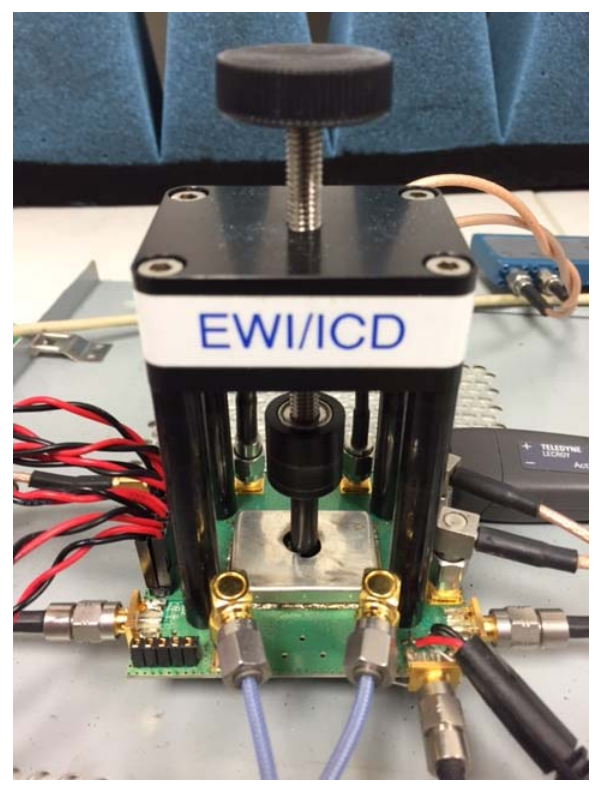

Fig. 6: A photograph of the measurement setup of the dual-channel OT. The package is inside the RF shield and the RF outputs are positioned perpendicular. pulling. A photograph of the measurement setup is shown in Fig. 6.

The output signals of channel 1 and 2 are summed by a power combiner, representing the signal the receiver would detect. This summed signal is measured by a spectrum analyzer. In Fig. 7 the sum of the output of channel 1 and 2 is plotted versus the BB phase difference, $\varphi_{1}-\varphi_{2}$, as generated by the AWGs. The measurements are carried out with an $f_{\mathrm{LO}}$ of $8 \mathrm{GHz}$ and $f_{\mathrm{BB}}$ of $1 \mathrm{MHz}$. Fig. 7 also shows the theoretic power-phase curves for a dual-element array having only gain mismatch between the two channels. Curves are shown for $1 \%$ and $10 \%$ gain mismatch. For our dual-channel OT chip, the total gain mismatch between the channels was $5 \%$. It is limited by the hybrids used in the measurement set-up.

The measurements indicate that a single-chip dual-channel OT can generate a beam pattern and can act as a BB beam former. It also shows that implementing two channels on-chip have a gain mismatch of $5 \%$.

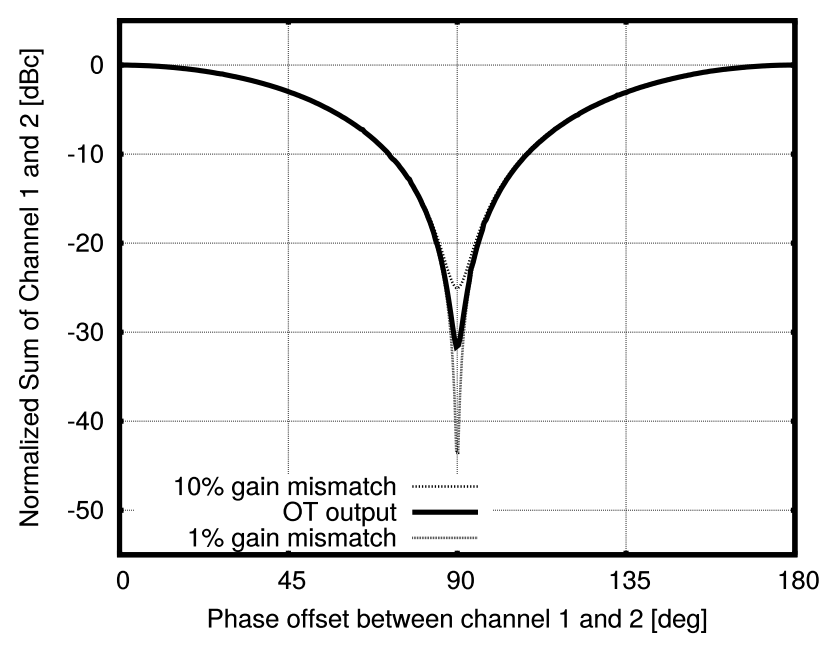

Fig. 7: Beam steering radiation pattern of two isotropic radiators.

\section{IMPACT OF OT-LIMITATIONS ON DDMA CAPABILITIES OF ARRAYS}

The impact of OT non-idealities on DDMA capabilities are derived from measurements on our dual-channel OT chip, assuming configuration $\mathrm{B}$ in Fig. 4, implemented almost similar to the way discussed in section III. In this set-up only one output of the dual-channel IC is connected to a spectrum analyzer, without a circulator, while the other is terminated to

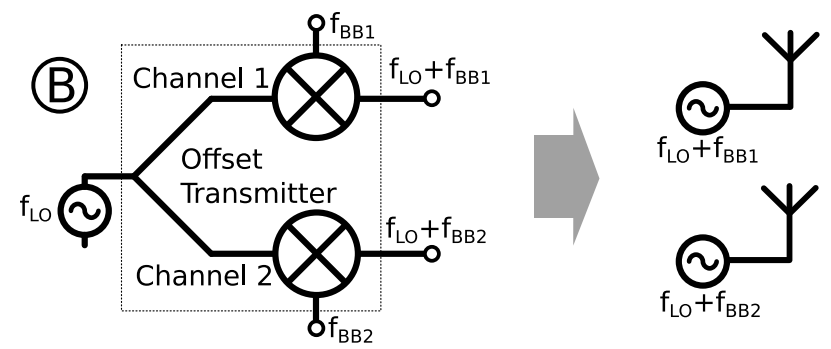

Fig. 8: Offset transmitter (left) used to implement DDMA (right); the resulting antenna pattern scans continuously for a fixed for $f_{\mathrm{BB} 1}$ and $f_{\mathrm{BB} 2}$. 


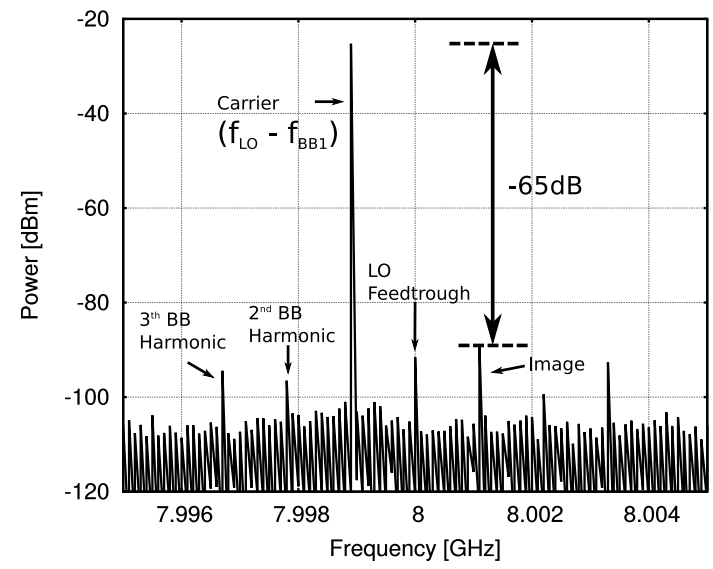

Fig. 9: The calibrated spectrum of channel 1, with channel 2 powered down. $f_{\mathrm{BB} 1}=1.2 \mathrm{MHz}$ and $f_{\mathrm{LO}}=8 \mathrm{GHz}$.

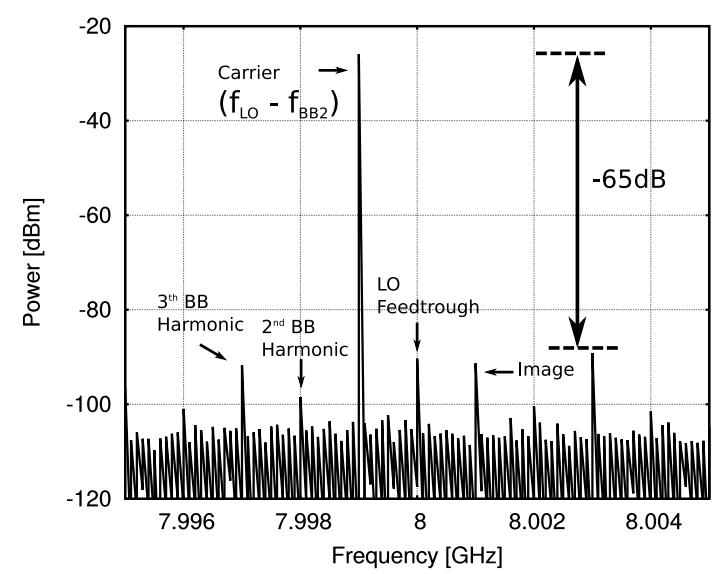

Fig. 10: The calibrated spectrum of channel 2, with channel 1 powered down. $f_{\mathrm{BB} 2}=1 \mathrm{MHz}$ and $f_{\mathrm{LO}}=8 \mathrm{GHz}$.

\section{$50 \Omega$ on-chip.}

Fig. 8 shows the simplified setup, in which the two OTs in a single IC create different frequencies from a common LO. Two external AWGs generate two BB signals For the measurement $f_{\mathrm{BB} 1}=1.2 \mathrm{MHz}$ and $f_{\mathrm{BB} 2}=1 \mathrm{MHz}$.

Each OT is calibrated individually using the method described in [7]. When the OT in channel 1 is calibrated, the OT in channel 2 is turned off by reducing the BB amplitude to zero, and vice versa. This way the strength of all in-band spurious responses is reduced. For this work, the spurs are reduced below $-65 \mathrm{dBc}$. The spectra of the calibrated channel 1 and 2 are shown in Fig. 9 and 10. Here, only the indicated channel is active.

After calibration, both OTs are powered up by setting the BB amplitude to the appropriate level. The output of channel 2 is measured with a spectrum analyzer and plotted in Fig. 11, with both channels active. It shows the dominant signal of $f_{\mathrm{LO}}+f_{\mathrm{BB} 2}$. At intervals of $f_{\mathrm{BB} 2}$ spurious components are visible, due to LO feed-through, IQ mismatch and non-linearities. Also the tones generated at the output of channel 2 are visible, which are attenuated by channel-to-channel isolation of $35 \mathrm{~dB}$. Other in-band spurs also appear due to inter-modulation effects between the two channels.

These measurements show that the single-chip dual-channel

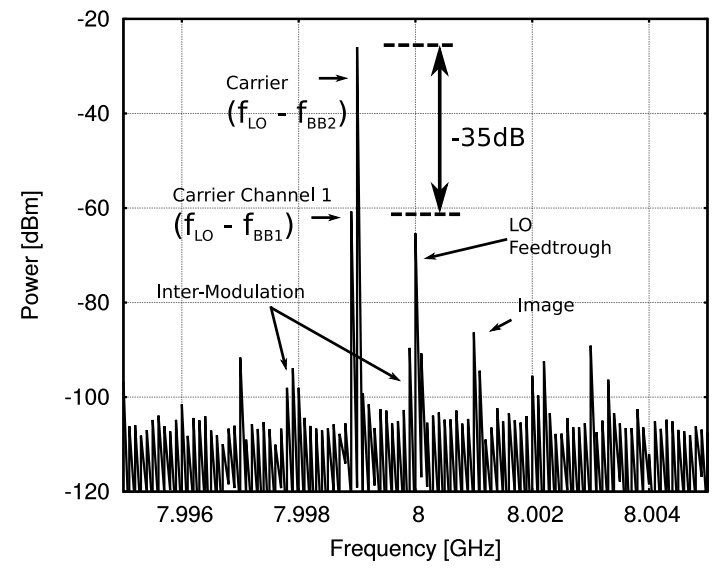

Fig. 11: The output of channel 2, with both on-chip OTs active.

OT is not suitable for DDMA purposes. The channel-tochannel isolation is too low and two active channels on a single chip give rise to pulling and inter-modulation errors, which cannot be compensated for a calibration algorithm that calibrates channels independently.

\section{CONCLUSIONS}

This work demonstrates the operation of the dual-channel Offset Transmitter in a practical and realistic setup. It shows the pros and cons of integrating two channels in a single IC. The single chip two-channel setup is suitable to generate two similar waveforms with a a static phase offset; making it a practical solution for a BB beam steering array. For DDMA waveforms, a single-chip solution lacks isolation at X-band frequencies, which results in (time dependent) pulling, crosstalk effects that show up as significant spurs in the OTs output signals.

\section{ACKNOWLEDGEMENTS}

The authors would like to thank E. Olieman, H. Westerveld, D.J. van der Broek, R. Struiksma, H. de Vries and G. Wienk for their support. NXP Semiconductors N.V. is acknowledged for donating silicon and providing models.

\section{REFERENCES}

[1] E. Fishler, et al., "Mimo radar: an idea whose time has come," in Radar Conference, 2004. Proceedings of the IEEE, 2004, pp. 71-78.

[2] S. Han, C. 1. I, Z. Xu, and C. Rowell, "Large-scale antenna systems with hybrid analog and digital beamforming for millimeter wave $5 \mathrm{G}$," IEEE Communications Magazine, vol. 53, no. 1, pp. 186-194, Jan 2015.

[3] E. Brookner, "MIMO radar demystified and where it makes sense to use," in Radar Conference (Radar), 2014 International, Oct 2014, pp. 1-6.

[4] D. Rabideau, "Doppler-offset waveforms for MIMO radar," in Radar Conference (RADAR), 2011 IEEE, May 2011, pp. 965-970.

[5] D. Fuhrmann, et al., "Waveform diversity and advanced signaling strategies for the hybrid MIMO phased array radar," in Waveform Diversity and Design Conference (WDD), 2010, pp. 283-283.

[6] D. J. Rabideau, "Hybrid mitigation of distortion in digital arrays," in Radar Conference, 2005 IEEE International, May 2005, pp. 236-241.

[7] J. C. J. G. Withagen, A. J. Annema, B. Nauta, and F. E. Vliet, van, "An 8-10 ghz upconversion mixer, with a low-frequency calibration loop resulting in better than $-73 \mathrm{dbc}$ in-band spurs," in Radio Frequency Integrated Circuits Symposium (RFIC), 2016.

[8] A. Hassanien and S. Vorobyov, "Phased-MIMO radar: A tradeoff between phased-array and MIMO radars," Signal Processing, IEEE Transactions on, vol. 58, no. 6, pp. 3137-3151, 2010. 